\title{
MAGNETICALLY DRIVEN JETS
}

\author{
R. V. E. LOVELACE \\ J. CONTOPOULOS \\ Department of Applied Physics \\ Department of Astronomy \\ Cornell University \\ Ithaca, New York 14853 \\ USA
}

\begin{abstract}
A simple but general theory for the formation and propagation of nonrelativistic jets is derived from the basic equations of ideal magnetohydrodynamics.
\end{abstract}

\section{INTRODUCTION}

Ordered magnetic fields in accretion disks may have a crucial role in producing the outflows or jets observed in many protostellar objects. In turn, the outflows may have a key role in removing angular momentum from the inner regions of the disk thereby facilitating the accretion of matter onto the star. Energetic bipolar outflows appear to be a common phenomenon associated with star formation (Lada 1985); in addition to the neutral outflows, there are narrow, high velocity jets of ionized material (Mundt 1985). In turn, flattened, rotating gaseous disks appear to be commonly associated with bipolar flows with the axis of the bipolar flow approximately aligned with the angular momentum axis of the disk (e.g., Boss 1987). A large number of rough theoretical models and ideas have been put forward to account for the observed bipolar flows. In one broad class of models, the twisting of a poloidal magnetic field threading the rotating star or disk acts to accelerate the bipolar flows (Draine 1983; Hanawa et al. 1988; Pudritz and Norman 1986; Uchida and Shibata 1985; Shu et al. 1988; and Konigl 1989). This class of models parallels the model put forward earlier by Lovelace (1976) and Blandford (1976) to explain the jets observed to emanate from the compact nuclei of active galaxies and quasars. In this model, the twisting of a poloidal magnetic field by a rotating conducting accretion disk acts to generate oppositely directed jets perpendicular to the disk. The jets carry away mass, angular momentum, and energy extracted from the disk - thereby - facilitating the accretion of matter onto the central object. In regard to bipolar flows the removal of angular momentum by the jets is of particular importance in view of the slow rotation rates of T-Tauri stars (Hartmann et al. 1986).

Only the magnetohydrodynamic (MHD) or 'field-line twisting' model appears as a likely universal model for the origin of jets in bipolar star-forming systems, in extra-galactic radio sources, as well as in other objects such as SS 433. The theory of the MHD origin of jets

R. Beck et al. (eds.), Galactic and Intergalactic Magnetic Fields, 337-340.

(c) 1990 IAU. Printed in the Netherlands. 
has come under increasingly intense study in recent years. Schematic models for the origin of MHD jets and outflows from disks and stars have been put forward by Blandford and Payne (1982) and by Sakurai (1985). Recent work has developed the theory of axisymmetric MHD to the point where detailed analytic calculations of jets are feasible (Lovelace et al. 1986; Lovelace 1987; Lovelace, Wang, and Sulkanen 1987; Camenzind 1987; Mobarry 1988; and Shu et al. 1988).

A recent and promising direction of the theoretical work on MHD jets from accretion disks is based on the envelope equations where one averages the different physical variables over the cross section of the jet at a given distance $\mathrm{z}$ from the equatorial plane (Lovelace 1987; Mobarry 1988; Koupelis 1988; Lovelace, Mobarry, and Contopoulos 1989 (denoted as LMC subsequently); Koupelis and Van Horn 1989). Here, we outline this approach.

\section{THEORY}

We use a cylindrical, inertial coordinate system $(r, \phi, z)$ with origin fixed at the central object and the $\mathrm{z}$ axis perpendicular to the disk. The outflows or jets are considered to be axi-symmetric $(\partial / \partial \phi=0)$ and stationary $(\partial / \partial t=0)$. The present treatment neglects relativistic corrections and dissipative effects.

The basic equations for the jets are those of ideal non-relativistic magnetohydrodynamics (MHD). In particular, the Euler equation is $\rho(\underline{v} \bullet \underline{\nabla}) \underline{v}=-\underline{\nabla} p+\rho g+\underline{J} \times \underline{B} / c$, where $\underline{v}$ is the flow velocity in the jet, $\rho$ is the jet density, $\mathrm{p}=\rho \mathrm{kT} / \mu$ is the kinetic pressure in the jet (with $\mu$ the mean particle mass), and $\mathrm{g}$ is the gravitational acceleration. As an approximation, we take $g$ to be given by the Newtonian field of the central object, that is, $g$ $=-\underline{\nabla} \Phi_{\mathrm{g}}$, with $\Phi \mathrm{g}=-\overline{\mathrm{G} M} /\left(\mathrm{r}^{2}+\mathrm{z}^{2}\right)^{1 / 2}$, where $\mathrm{G}$ is the universal gravitational constant, and $M$ is the mass of the central object.

We are mainly interested in the axial ( $\mathrm{z}$ ) variations of the jet parameters such as the axial and azimuthal flow speeds and the magnetic field components. Therefore, we assume a simple self-similar axial variation of the transverse $(r)$ dependences:

$$
v_{r}(r, z)=\left[\frac{r}{a(z)}\right]\left[\frac{d a(z)}{d z}\right] v_{z}(z) ; v_{\phi}(r, z)=r \Omega(z) ; v_{z}(r, z)=v_{z}(z) ; \rho(r, z)=\bar{\rho}(z) f(\alpha) .
$$

Here, $a(z)$ is the jet radius; $\Omega(z)$ is the rigid body rotation rate of the jet at an axial distance $z ; \quad \bar{\rho}(z)$ is the average density of the jet at $z ; \alpha \equiv[r / a(z)]^{2}$ is a useful dimensionless measure of radial distance within the jet: and $f(\alpha) \geq 0$ is a dimensionless mass-density "profile function" normalized so that its integral $(0,1)$ is unity. For specificity we take $f(\alpha)=(n+1) \alpha^{n}$. Thus $n=0$ corresponds to a uniform density, while $n \geq 1$ corresponds to a distribution peaked at the jet's edge.

The mass-flow in the jet across any $z=$ const. surface is a constant,

$$
\dot{\mathrm{M}}=\int_{\mathrm{z}=\text { const }} \mathrm{d}^{2} \times \rho \mathrm{v}_{\mathrm{z}}=\pi[\mathrm{a}(\mathrm{z})]^{2} \rho(\mathrm{z}) \mathrm{v}_{\mathrm{z}}(\mathrm{z})=\text { const. }
$$

The magnetic field obeys the "frozen-in" field so that we may take 
$B_{r}=\left[\frac{r}{a(z)}\right]\left[\frac{d a(z)}{d z}\right] B_{z} ; B_{\phi}(r, z)=\frac{\alpha^{1 / 2}[\omega(z)-1] B_{i}(\alpha)}{V_{z}(z) A(z)} ; B_{z}(r, z)=\frac{B_{i}(\alpha)}{A^{2}}$

for $\alpha<1$, whereas for $\alpha>1$ we assume $\underline{B}=0$. Here, $V_{z}(z) \equiv v_{z}(z) / v_{0}$ is a dimensionless axial jet speed, where $v_{0} \equiv \Omega(z=0) a_{i}$ is the initial azimuthal velocity of the jet. $\omega(\mathrm{z}) \equiv \Omega(\mathrm{z}) \mathrm{a}_{\mathbf{i}} / \mathrm{v}_{0}$ is the dimensionless rotation rate of the jet with $\omega(\mathrm{z}=0)=1$. Equation $(2)$ assumes that $\mathbf{v}_{\mathrm{z}}=(\mathrm{z} \rightarrow 0) \approx 0$. Because the magnetic field vanishes outside of the jet so that jet collimation is due to the gravitational field of the central object. [Modifications can easily be made to account for magnetic and pressure fields external to the jet.]

We consider field profiles of the form $B_{i}(\alpha)=B_{0} \alpha m$, with $m \geq 0$. $B_{o}$ provides a useful normalization value for the magnetic field for $z>0$. For $m=0$ the axial field is uniformly distributed, whereas for $m \geq 1$ it is concentrated near the jet's edge.

An equation for the conservation of the axial momentum flow of the jet can be readily obtained (LMC) by integrating the general momentum conservation equation over a volume of the jet between $\mathrm{z}$ and $\mathrm{z}+\mathrm{dz}$. The result is:

$$
\frac{\mathrm{d}}{\mathrm{dZ}} \mathcal{F}_{\mathrm{p}}=\frac{\mathrm{F}_{\mathrm{z}}}{\mathrm{V}_{\mathrm{z}}}
$$

where $\mathrm{Z} \equiv \mathrm{z} / \mathrm{a}_{\mathrm{i}}$ is a dimensionless axial distance; $\mathrm{F}_{\mathrm{z}}$ is the axial gravitational force on the jet matter within $\mathrm{z}$ to $\mathrm{z}+\mathrm{dz}$; and $\mathcal{F}_{\mathrm{P}}$ is the dimensionless axial momentum flow of the jet,

$$
\left(\mathrm{a}_{\mathrm{i}} \mathrm{B}_{\mathrm{o}}\right)^{2} \mathcal{F}_{\mathrm{P}}=\int_{\mathrm{z}=\mathrm{const}} \mathrm{d}^{2} \mathrm{x}\left(\mathrm{T}_{\mathrm{p}}^{\mathrm{zz}}+\mathrm{T}_{\mathrm{f}}^{\mathrm{zz}}\right)=\dot{\mathrm{M}}\left(\mathrm{V}_{\mathrm{z}}+\frac{\tau}{\mathrm{V}_{\mathrm{z}}}\right) \mathrm{v}_{0}+\frac{1}{4} \int_{0}^{\mathrm{a}} \operatorname{rdr}\left[\mathrm{B}_{\mathrm{r}}^{2}+\mathrm{B}_{\phi}^{2}-\mathrm{B}_{\mathrm{z}}^{2}\right]
$$

Here, $T_{p} i j$ is the stress tensor for the matter, and $T_{f} i j$ is that for the magnetic field; $\tau \equiv \mathrm{kT} /\left(\mu \mathrm{v}_{0}{ }^{2}\right)$ is the dimensionless jet temperature. In view of equations (11) and (12), we have

$$
\mathcal{F}_{\mathrm{P}}=\mathcal{F}_{\mathrm{M}}\left(\mathrm{V}_{\mathrm{z}}+\frac{\tau}{\mathrm{V}_{\mathrm{z}}}\right)+\frac{1}{2} \mathcal{F}_{0}\left[\left(\frac{\mathrm{A}^{\prime}}{\mathrm{A}}\right)^{2}+\left(\frac{\omega-1}{\mathrm{~V}_{\mathrm{z}}}\right)^{2}-\frac{\eta}{\mathrm{A}^{2}}\right]
$$

where $\mathcal{F}_{M}$ is the dimensionless mass flow rate, $A^{\prime}=\mathrm{dA} / \mathrm{dZ}$, and $\mathcal{F}_{0}$ and $\eta$ are dimensionless constants. For $B_{i}(\alpha)=B_{0} \alpha^{m}, \mathcal{F}_{0}=1 /[8(m+1)]$ and $\eta=$ $2(\mathrm{~m}+1) /(2 \mathrm{~m}+1)$.

The gravitational force $F_{z}$ in equation (13) depends of course on the distribution of density with $\mathrm{r}$ in the jet (LMC). For example, if the density is peaked near the jet's edge, $F_{z}=-Z \delta\left(A^{2}+Z^{2}\right)-3 / 2$ where $\delta=G M \mathcal{F}_{M} /\left(a_{i} v_{0}{ }^{2}\right)$ is a dimensionless measure of the strength of the gravitational force. Notice that for a non-magnetic, non-relativistic Keplerian disk $\mathrm{GM} /\left(\mathrm{v}_{0}{ }^{2} \mathrm{a}_{\mathbf{i}}\right)=1$.

In addition to equation (13), equations for the conservation of angular momentum and energy can be similarly derived. Together these equations allow one to solve for the jet velocity, radius, rotation rate and temperature from $\mathrm{Z}=0$ to $\infty$. The jet solutions are however constrained by the fact that the mass, angular momentum, and energy carried by the $\pm \mathrm{z}$ jets must equal the loss of these quantities by the disk. Figure 1 illustrates a self-consistent jet/disk solution. 


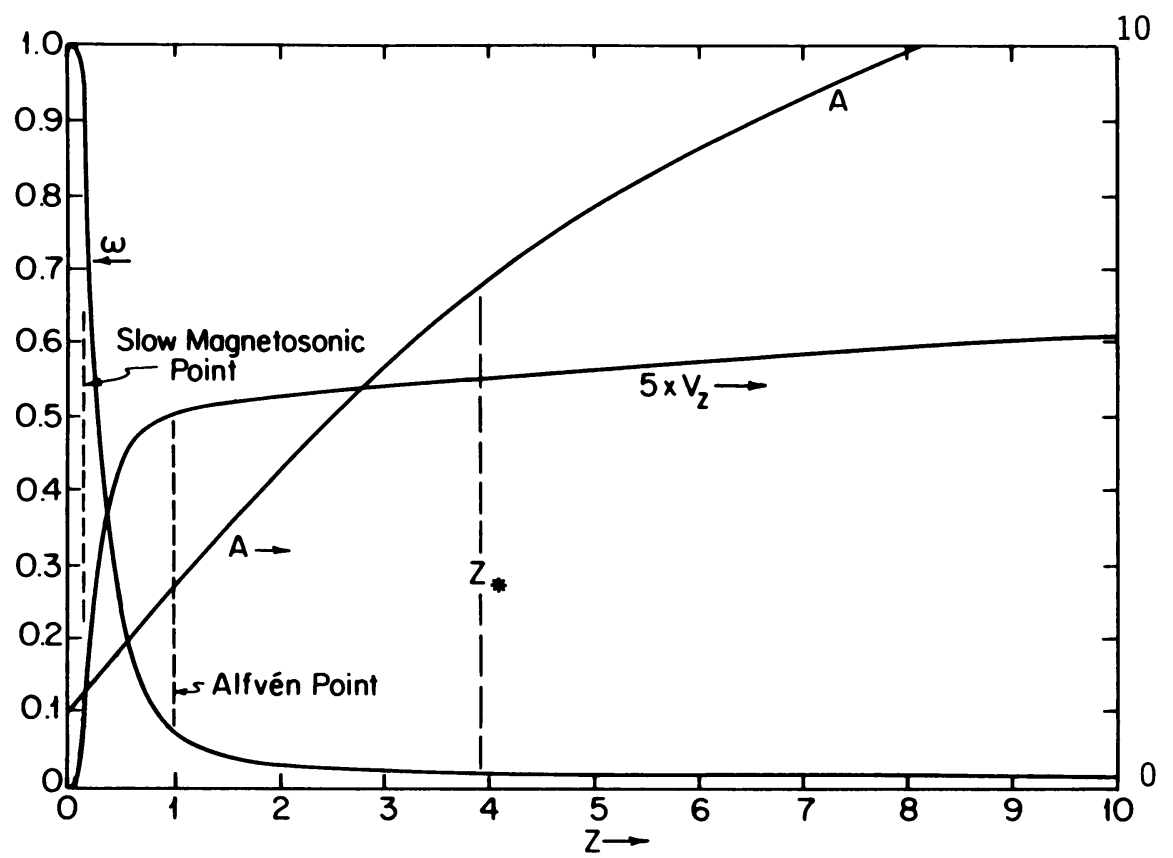

Figure 1. The axial variation of the jet variable close to the central object.For $Z<Z_{*}$ the jet is isothermal whereas for $\mathrm{Z}>\mathrm{Z}_{*}$ it is adiabatic. The fast magnetosonic point is at $\mathrm{Z}=\infty$.

\section{REFERENCES}

Blandford, R. D., 1976, M. N. R. A. S. 176, 465.

Blandford, R. D., and Payne, D. G., 1982, M. N. R. A. S. 199, 883.

Boss, A. P., 1987, Ap.J. 316, 721.

Camenzind, M., 1987, Astron, and Astrophys. 184, 341.

Draine, B. T., 1983, Ap. J. 270, 519.

Hanawa, T., Kamahori, H., Maruyama, T., and Shibata, K., 1988, Pub. Astron. Soc. of Japan 40, 6.

Hartmann, L., Hewett, R., Stahler, S., and Mathieu, R. D., 1986, Ap. J. 309, 275.

Konigl, A., 1989, Ap. J, 342, 208.

Koupelis, T., 1988, Ph.D. Thesis, University of Rochester.

Koupelis, T., and Van Horn, H. M., 1989, Ap. J. 342, 146.

Lovelace, R. V. E., 1976, Nature 262, 649.

Lovelace, R. V. E., 1987, in "Magnetic Fields in Extra-Galactic Objects", E. Asseo and D. Gresillon, Eds. (Editions de Physique, France), p. 223.

Lovelace, R. V. E., Mehanian, C., Mobarry, C. M., and Sulkanen, M. E., 1986, Ap. J. Suppl. $62,1$.

Lovelace, R. V. E., Mobarry, C. M., and Contopoulos, J., 1989, in "Accretion Disks and Magnetic Fields in Astrophysics", G. Belvedere (ed.), (Kluwer Academic Publishers), p. 71.

Lovelace, R. V. E., Wang, J. C. L., and Sulkanen, M. E., 1987, Ap. J. 315, 504.

Mobarry, C. M., 1988, Ph.D. Thesis, Cornell Unviersity.

Mundt, R., 1985, in "Protostars and Planets II", D. C. Black and M. S. Mathews, Eds. (Univ. of Arizona Press) p. 414.

Pudritz, R. E., and Norman, C. A., 1986, Ap. J. 301, 571.

Sakurai, T., 1985, Astron, and Astrophys. 152, 121.

Shu, F. H., Lizano, S., Ruden, S. P., and Najita, J., 1988, Ap. J. 328, L19. 